\title{
The Algorithm for Extracting Elements of National Costume Based on Region Growing
}

\author{
Deqiang Yang, Jianhou Gan, Bin Wen and Tianwei Xu \\ School of Information Science and Technology, Yunnan Normal University; Key Laboratory of Educational Information for \\ Nationalities, Ministry of Education of China, Kunming 650500, China
}

\begin{abstract}
It is difficult to effectively extract costume elements from the national costume image because the structure of costume images is complex and the gray distribution is irregular. Therefore, in this paper, a novel algorithm was proposed for the extraction of the elements using the mathematical morphology method for element internal filling and external pruning. An improved algorithm for region growing image segmentation was proposed to dynamically calculate the mean and variance of seed regions as growth parameters. According to the elements of the gray dispersion, the seeds were divided into three kinds of them to enter the target image which need to be extracted. Different types of seeds played different roles in the growth process. The target element were gradually obtained with each seed growth. The experimental results show that the relatively complete and accurate elements of the costume can be extracted by the algorithm.
\end{abstract}

Keywords-morphology gradient; region growing; costume element; gray discretization algorithm

\section{INTRODUCTION}

The protection and development of minority costumes is one of the important parts of the inheritance and development of national culture. The production and preservation of the existing national dress patterns with digital multimedia technology to achieve the effective collection of the national costume culture, is an important work to promote the digital construction of minority costumes[1]. The elements in the national dress with many exquisite workmanship and fashion and with complex structure is the most important expression and embody for the national dress features. Some of which is a symbol of national culture, and the others are the essence of art creation. If the elements can be extracted from the clothing image in full dress, they can be used to enhance the identification and understanding, protection and inheritance of the nation clothing. Many elements of pattern have the higher values for reprocessing, utilization and research.

The existing image segmentation algorithms for extractions of elements in costume image are based on threshold method, edge based method, region based method and/or theory based method. Threshold segmentation includes single threshold segmentation and multi-threshold segmentation. The single threshold segmentation means that the image is simply divided into two parts, and the multi-threshold segmentation means that the image is divided into many parts according to multiple threshold and it is mainly for two value image. Median of two peak value from histogram or iterative threshold value is chosen as a threshold value. A typical algorithm with a watershed is to allow the value of the two low-lying areas continue to expand outward. When the gray value of the two regions of the intersection, the threshold is needed. Regional growth is a process of increasing seed. Starting from the initial seed given by growth, the seed is grown to adjacent areas under a certain growth criteria, and then the pixels with similar properties are added to the seed region until no seeds with similar properties can be found. As a result, many image segmentation regions are obtained. The three key factors of the region growing method is: 1) to choose the suitable seed points; 2) to determine the criterion that can combine the pixels in the growth process; 3 ) to set the conditions that can stop the growth. The advantages of the region growing method is simple and efficient, especially suitable for simple and strongly regular image. But it is its disadvantages that human interaction is needed to obtain the seed point. At the same time, the method is also sensitive to noise, easy to cause over-segmentation or under-segmentation. In order to overcome these shortcomings, some scholars put forward some improved methods, for example, the methods based on statistical information of a certain region[2], the method of combining region growing with graph cut algorithm[3-4], the method based on learning framework[5-6], and the algorithm based on laplacian local feature description[7]. There are some limitations with these algorithms. Using the existing algorithms, the extracted costume elements are often incomplete, not integral, or not accurate, because of the particularity of national dress, including complexity of structure of costume elements, large changes in gray level, weak continuity. And thus, multi elements often adhere to each other together so that the complete and accurate costume elements cannot be extracted.

This paper made an observation and statistical analysis of a large number of clothing images and costume elements. The results show that the gray change of clothing elements is particularly large. The gray values can change from 0 to 255 . There are no concentration of gray level, no rules, and no obvious boundary between elements and elements. Furthermore, adhesion is common, and structure is complex. Minor information is also more. Thus, the extracting effect is very poor when using a single fixed threshold. The desired effect cannot be obtained under most of the image segmentation algorithms. In view of these situations, a dynamic threshold region growing algorithm is proposed. Firstly, using image processing technology of mathematical morphology, the pretreatment for costume image are conducted, including that the holes in costume elements are filled, and that the types of adhesion between elements and between element and background are trimmed according 
morphological gradient in costume image. The two methods, i.e., artificial selection and automatic global search, are used to generate an initial seed. The algorithm adopts the mean of seed region as the standard and variance as threshold. The standard and the threshold are dynamically adjusted. At the same time, according to the pixel gray dispersion, the seeds which are put into the target area is divided into three categories so as to let them play a different role in the follow-up of the regional growth process. The target elements are obtained. The second part of the present paper is the introduction about image preprocessing with technology of mathematical morphology. The third part is the description of the region growing algorithm based on dynamic threshold.

\section{IMAGE PREPROCESSING WITH MATHEMATICAL MORPHOLOGY}

For extraction of national costume elements, there are two difficulties in segmentation element. The first of them is that the costume elements which need to be extracted from the costume design can have a certain correlation with the surrounding image at the aspect of the gray. Sometimes it is difficult to distinguish them with the human eye. Some small elements have a lot of convergence with the background. Thus, some regions which do not belong to the target areas can be entered into the target areas by the algorithm. Conversely, the second is that some necessary regions can be rejected to enter into the target. There is some discontinuous and open places in the region, so that some elements belonging to the target part is rejected to enter into the target. Therefore, before conducting the regional growth algorithm, the images are properly pruned and filled. To solve the problems, the image is preprocessed using mathematical morphology[8] method. Thus, the inner region breakpoints are filled; and the unnecessary external adhesion is pruned.

The basic idea of morphological image processing is to move a structure element at the range of image, and it is conducted by the way of convolution. Structure elements can be of any size. However, according to the features of the image which needs to be treated, the structure operator with the same characteristics can be constructed to improve the effect of image processing. The basic operations of the morphology are the expansion and the corrosion. They are combined to produce a variety of practical algorithms, and thus to better maintain the details, the shape and the edge of the image. The formulas for corrosion, expansion and gradient operations used in this paper are as follows, from (1) to (3).

$$
\begin{aligned}
& (f \Theta g)(x)=\max \left\{y: g_{x}+y<f\right\} \\
& (f \oplus g)(x)=\min \left\{y: g_{x}+y>f\right\} \\
& \mid G R A D(f)=(f \oplus g)-(f \Theta g)
\end{aligned}
$$

In the formulas, f represents the image to be processed and grepresents the structure operator. According to the image for extracting costume elements, the suitable structure operator is set, and the internal holes in the image are filled using the expansion and erosion operation. In the following steps, the formula (3) is used to calculate the morphological gradient of image, and to cut adhesion between elements and between elements and background. Therefore, the good edges of the dress elements for extraction and other elements can be maintained so as to provide an ideal image for the subsequent step of region growing algorithm.

The extracting effects will be good when there are the large differences between the costume elements and between the elements and the background. But if the gray level difference between the clothing elements is small, the results from implementation of the algorithm will be affected at some degree. To solve the question, the gray discrete function can be designed according to the image histogram before the implementation of the regional growth algorithm, so as to gather the image gray level to the peak of gray level. Figure 1 is a graph from a gray discrete function used in the experiment.

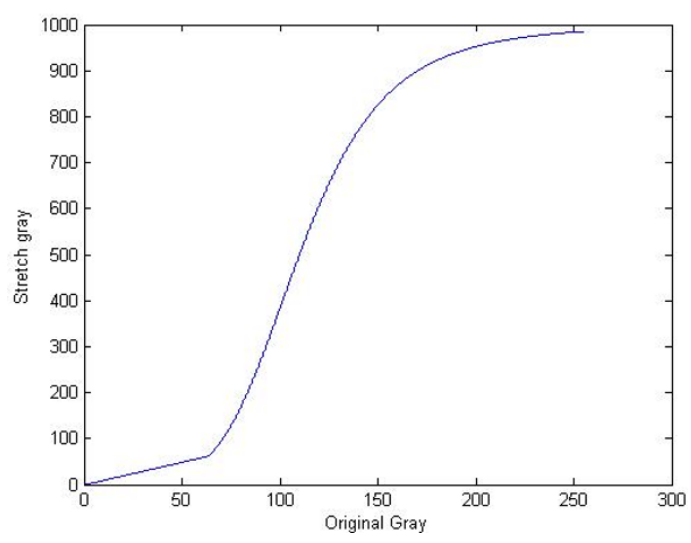

FIGURE I. FUNCTION OF GRAY DISCRETE

\section{REgION GROWING AlgORITHM WITH DYNAMIC THRESHOLD}

There are two key problems to be solved in the image segmentation algorithm with the region growing method. One of them is the seed selection, and the other is the threshold selection for the target image.

\section{A. Algorithm for Seed Selection}

A costume element is an area with similar gray level in the image. The two methods can be used to set a seed. As for the first method, a pixel or a small pixel area is manually specified as the initial seed, and then the costume element can be quickly extracted by the subsequent algorithm. For the two method, the global searching method is used. A random point in the image will be chosen as a start point. The point and the area of its surrounding which is not segmented with the point are used as an initial seed. Subsequently, the pixels which meet the conditions of the above is added to the seed set, and the pixel which do not meet the conditions is chosen as a new segmented region, until no new pixel is added to the present seed set. As a result, the first block, area1, is obtained. Then, similarly, from the rest of the area waiting to be segmented, one pixel or the surrounding part which is not segmented are taken as a seed, and the previous search is repeated to get the second partition area2. This cycle continues until all the 
regions of the original image are divided. The steps of algorithm for automatic generation of seed are as follows:

a) In the image, any point and its part of area which is not segmented are chosen to form the current seed set;

b) The region growing algorithm in this paper is called to expand the seed region;

c) When the current seed area is no longer expanded, the segmentation of the current region is completed.

d) If any region which is not segmented still exist in the image, Steps a)-c) are repeated, or it is the end of the algorithm.

The two methods are used in the experiment. The algorithm for automatic generation of seeds using iterative methods, gradually gets the desired target elements. On the other hand, by the manual way, the desired target elements can be quickly extracted.

\section{B. Region Growing Algorithm}

The area with $3 * 3$ or $5 * 5$ pixels can be used as an initial seed set to calculate the mean value of the seed region, Mean, as a standard gray value of the seed. Then the standard deviation (StD), maximum (Max) and minimum (min) of the seed region are calculated, which are the standards to determine whether a pixel belongs to the region. The gray dispersion of the pixels in the region is calculated based on the formula (4). With the method for region growth of seeds, the qualified pixels are searched within the region by 8 fold of the seed region or more, and continually added to the seed region. When the new pixel is added to the seed region the mean, standard deviation, maximum and minimum values of the seed area are updated. These steps are repeated until no new pixels are added to the seed region. Thus, the algorithm ends, and the segmented image is obtained.

$$
\text { disp }=\mid f(x, y)-\text { Mean } \mid
$$

Many studies have been done on threshold selection in the literatures, and the experimental results is in common. For the image with the costume elements, the differences in its gray levels is large, and the others are relatively small. Thus, if a fixed threshold only is used to divide, it is difficult to determine the threshold. But, if set by experience, the algorithm is not universal.

$$
\begin{gathered}
\mathrm{M}=\frac{1}{\mathrm{~N}} \sum_{f(x, y) \in \mathrm{D}}(f(x, y)-\mathrm{M}) \\
\sigma=\left(\frac{1}{\mathrm{~N}} \sum_{f(x, y) \in \mathrm{D}}(f(x, y)-\mathrm{M})^{2}\right)^{\frac{1}{2}}
\end{gathered}
$$

Using the formulas (5) and (6), the mean and standard deviation of the seed area are calculated. The mean value of the pixels in the seed region is selected as the standard value of the seed. Additionally, standard difference in the seed set reflects the discrete degree of gray levels in costume elements. The obvious differences in the variances in different costume elements can be found easily. Therefore, it is appropriate that the standard deviation is used as the threshold value to determine whether a new pixel enter the target image. And the algorithm is also self-adaptive at some degree. The setting of self-adaptive threshold makes the method is more universal for image segmentation. When the new pixels are entered into the seed region, the mean and standard deviation of the seed region are recalculated.
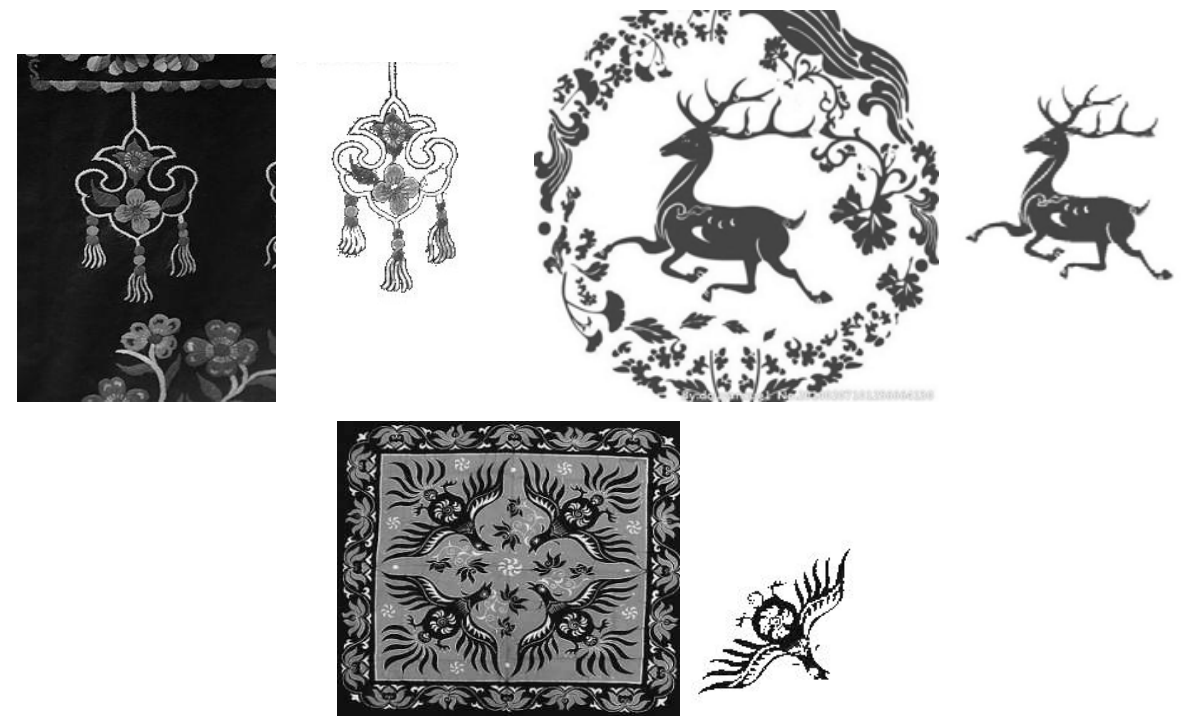

FIGURE II. THE EXPERIMENT EXAMPLE OF THIS PAPER ALGORITHM

If the discrete degree of gray levels (disp) of a new pixel is less than the standard deviation, the pixel in good condition will be entered into the seed set. Such pixel which meets this kind of condition and be entered into the seed set, is called as the first class of new seed.

There are some strict requirements for the first kind of 
seeds. But, at the actual situation, some pixels do not meet the condition, but it is a part of the costume elements. Based on the observation, the gray value of the pixels in such regions is between the maximum and minimum of the gray values of the seed region. Therefore, the region does belong to the target image, and the pixels in the region should also be entered into the seed region, being known as the second class of seeds.

Due to noise or sampling, or the structure characteristics of the costume elements, some isolated points or areas exists in the image. Its discrete degree of gray levels is far greater than the dynamic threshold, but it belongs to the target image. At this point, the average value of the region around the $3 * 3$ or $5 * 5$ is taken as the decision value for this point, and the discrete degree of the gray level is calculated. If the threshold condition is satisfied, the pixel can also be added to the seed region, which is called as the third kind of seeds. The third class of seed point may be caused by the noise in the image or by the edge pixels of the target image. So, when the third kind of seed points are entered into the seed region, the mean, variance, maximum and minimum of the values of the seed region are not updated.

After a pixel was added to the seed region, it is still necessary to search for the target pixels which meet the conditions, within the 8 areas around the added pixel. If the condition is satisfied, these pixels are entered into the seed region, and the same search must be still conducted until there is not a new pixel into the seed region. During the experiment, the queue is used to finish the process. In addition, a large discontinuity structure may exist in some clothing elements, or there is a problem of over-segmentation. At such situation, it is necessary to properly increase the searching radius, such as 24 points in the vicinity of the seed points to search for qualified seed points.

The algorithm is described as follows:

a) The seed area is selected, added to the seed area (D), added to the target image area, and added to the search Queue. The mean, standard deviation, maximum and minimum of the seed region are calculated.

b) If Queue is empty, the algorithm is ended; or else it is non-empty, the first element in the Queue is out of the team. Then, a new element is searched in its $\mathrm{R}$ field by following $c$ ). $\mathrm{R}$ is the search radius.

c) Comparing the gray value of the current element (the pixel which is being tested) with the average gray value, if the absolute value of the difference between them is less than the standard deviation the current pixel is added to the seed area, while being added to D and Queue.

d) If a pixel does not meet the conditions of c), namely, the absolute value of the difference is larger than the standard deviation, its gray value will be compared whether it is between max and min. If yes, the pixel is added to the seed region DS. At the same time, this pixel, as the second seed point, is added to the objective image region $\mathrm{D}$ and the queue Queue. e) If step c) and $d$ ) are not satisfied, the mean value of the $3 * 3$ field around the pixel is taken to test whether the pixel satisfies the conditions in Steps 3 or Step4. If yes, as the third seed points, it is added to the objective image region $\mathrm{D}$ and the queue Queue, but is not added to the seed region DS.

f) For the generated seed region DS, the mean M, standard deviation $\sigma$, maximum Max and minimum min are recalculated. Then, $b$ ) is repeated.

\section{EXPERIMENTAL EXAMPLE}

In the algorithm, the threshold of the region growth is determined by the standard deviation of the gray value of the pixel in the target image, and is updated when a new pixel is added to the seed region. In general, it is only necessary to search for the new seed point from the 8 areas around the qualified seed point. But for some image, because of the light or the structure of the image itself, there are some internal discontinuous structure. In this case, it is necessary to search from the 24 areas around the seed point. It can be avoided that the discontinuous structure is excluded from the target image. Over segmentation maybe exist when using image segmentation algorithm. To solve this problem, after the end of the algorithm, the segmented blocks can be appropriately merged to achieve the purpose of reasonable segmentation according to the average of each block and the adjacent distance

\section{ACKNOWLEDGMENT}

Supported by the National Natural Science Foundation of China (61262071, 61562093, 61462097); Supported by the basic research program of Yunnan province (2016FA024).

\section{REFERENCE}

[1] Chunyin Qi. Research on Chinese ethnic culture and art[M]. Beijing: Nationalities Publishing House, 2012, 9.

[2] Law T Y, Heng P A. Automated Extraction of Bronchus from 3DCT Images of Lung Based on Genetic Algorithm and 3D Region Growing[C] Proc of SPIE, 2000: 9062916.

[3] Shaoping $\mathrm{Xu}, \mathrm{Xiao-ping} \mathrm{Liu.} \mathrm{A} \mathrm{fast} \mathrm{image} \mathrm{segmentation} \mathrm{algorithm} \mathrm{based}$ on region maximal similarity[J]. Journal of Optoelectronics Laser. 2013, 24(5): 990-998.

[4] xin-zheng Xu, Shi-fei Ding, Zhong-zhi Shi. New Theories and Methods of Image Segmentation[J]. Acta electronica Sinica, 2010,38( 2): 76-82.

[5] Guo-Bao Hui, Yi-Fei Tong, Dong_bo Li. Image Features Matching Based on Improved Patch Similarty Learning Framework[J]. Chinese Journal of Computers, 2015,38(6): 1148-1161

[6] Peng B, Zhang L, Zhang D, Image segmentation by iterated region merging with localized graph cuts[J], Pattern Recognition,2011,44(10): 2527-2538.

[7] Malekesmaeili M, Ward R, Fatourechi M, A fast approxi-mate nearest neighbor search algorithm in the hamming space[J], IEEE Transactions on Pattern Analysis and Machine Intelligence, 2012, 34(12): 2481-2488

[8] Serra J. Image analysis and mathematical morphology [M]. New York: Academic Press, 1982 